\title{
Epidermal growth factor reduces multiorgan failure induced by thioacetamide
}

\author{
M E Caballero, J Berlanga, D Ramirez, P Lopez-Saura, R Gozalez, D N Floyd, \\ T Marchbank, R J Playford
}

\begin{abstract}
Background-Multiorgan failure is a severe life threatening state where present therapeutic approaches are suboptimal. Epidermal growth factor (EGF) is a potent stimulant of repair in in vitro and in vivo models. We therefore examined its potential beneficial effect in reducing mortality and injury induced by the noxious agent thioacetamide (TAA).

Methods-Mice (20 per group) were fasted overnight and received a single intraperitoneal dose of human recombinant EGF at 10 or $30 \mu \mathrm{g} / \mathrm{kg}$ or saline (control). Either 30 minutes before or after EGF, all animals also received TAA $(40 \mathrm{mg} / \mathrm{kg}$ intraperitoneally). Twenty four hours later, surviving animals were killed, tissues collected, and degree of organ injury assessed.

Results-Fifty per cent (10/20) of control animals died within the first 24 hour period. Mortality was almost completely prevented by the higher dose of EGF whether given before or after TAA $(p<0.01)$ and was reduced by about $50 \%$ with the lower dose of EGF. In control animals, the entire length of the jejunum and ileum had necrosis with or without mucosal denudation. In contrast, necrosis affected only about $10-20 \%$ of the total length in EGF treated groups (both $\mathbf{p}<0.01$ $v$ control). Control animals showed marked glomerular tuft collapse, interstitial haemorrhage, and increased plasma creatinine levels. These effects were significantly reduced in animals given EGF (30 $\mu \mathrm{g} / \mathrm{kg} ; \mathrm{p}<0.01)$. All groups showed similar changes in liver histology (centrilobular necrosis) and alanine transaminase levels (10-fold increase).

Conclusions-Although EGF did not prevent the hepatotoxicity associated with TAA, it reduced mortality, renal injury, and gastrointestinal damage. These studies provide preliminary evidence that EGF may be a novel approach for the prevention and/or treatment of multiorgan failure.

(Gut 2001;48:34-40)
\end{abstract}

Keywords: gastrointestinal damage; nephrotoxicity; liver injury

Professor R J Playford, Gastroenterology Section, Imperial College School of Medicine, Hammersmith Hospital Campus, DuCane Rd, London W12 0NN, UK. r.playford@ic.ac.uk

Accepted for publication 18 July 2000

Multiple organ failure (MOF) is a severe life threatening condition which usually occurs as a result of major trauma, burns, or fulminant infections. Whatever the initiating event, once established, MOF has a high mortality (up to
$80 \%){ }^{1}$ The pathophysiological mechanisms underlying this condition are unclear although important contributory factors probably include hypoxia, increased intestinal permeability, bacterial translocation, endotoxaemia, and uncontrolled systemic inflammatory responses (see Nguyen and colleagues ${ }^{2}$ ).

Novel therapies that reduce the risk of patients developing MOF have direct clinical potential. Many of the patients who progress to MOF while an inpatient can be identified at an early stage, often at the time of admission, based on the severity of initial injury, for example, percentage of skin surface area affected in burns patients. There is therefore a potential therapeutic window where agents such as epidermal growth factor (EGF) could be administered prior to the development of the full MOF syndrome.

Thioacetamide (TAA) has been used extensively as an hepatotoxin to establish animal models of acute and chronic liver injury. ${ }^{34}$ Its injurious effects have been attributed, in part, to the biotransformation of TAA to the TAA sulphone and sulphoxide forms which react extensively with proteins, resulting in their denaturation. ${ }^{56}$ Although less well studied, administration of TAA can also result in damage to the kidney (particularly the proximal tubule $^{7}$ ) and gastrointestinal tract, ${ }^{8}$ suggesting that administration of TAA might be a useful model of multiorgan failure.

EGF is a 53 amino acid peptide produced by the salivary glands and Brunner's glands of the duodenum. It is a potent stimulant of proliferation and healing of the gastrointestinal tract, acting as a cytoprotective agent, "stabilising" cells against noxious agents such as indomethacin. ${ }^{9}$ In addition, we have recently shown that EGF can prevent hepatic injury induced by carbon tetrachloride. ${ }^{10}$ There is therefore interest in its potential clinical value for the treatment of human gastrointestinal and hepatic disease. We now report our studies examining whether EGF can influence the degree of renal, hepatic, and gastrointestinal injury caused by the noxious agent TAA.

\section{Materials and methods}

ANIMALS

Adult male OF-1 male mice (20-22 g) were housed individually in wire bottomed cages and allowed free access to water and commer-
Abbreviations used in this paper: EGF; epidermal growth factor; hrEGF, human recombinant EGF; TAA; thioacetamide; $\mathrm{MOF}$, multiple organ failure; ALAT, alanine aminotransferase. 
cial chow. Animals were acclimatised for seven days before experiments, which were performed according to local regulatory guidelines.

\section{CHEMICALS}

Human recombinant (hr) EGF, expressed in Saccharomyces cerevisiae, was purchased from HeberBiotc SA (Havana, Cuba). Prior to administration, hrEGF was diluted in $0.9 \%$ saline and sterilised via passage through a 0.22 $\mu \mathrm{m}$ filter. TAA was obtained from Sigma Chemical Co. (St Louis, Missouri, USA) and diluted in saline. All solutions were freshly prepared before each experiment.

BIOCHEMICAL ASSAYS

Serum levels of alanine aminotransferase (ALAT) were measured colorimetrically, ${ }^{11}$ according to the supplier's instructions (Hoffman LaRoche, Basel, Switzerland). Serum levels of creatinine were measured according to the manufacturer's instructions (Reactivos SPINREACT SA, Barcelona, Spain).

EXPERIMENTAL PROTOCOLS

Mice were fasted for 16 hours before commencement of all experiments

Preliminary studies

A series of preliminary experiments were performed examining the effect on survival of various doses of TAA given alone. Groups of 10 mice were given $10,20,40$, or $60 \mathrm{mg} / \mathrm{kg}$ of TAA (single intraperitoneal injection) and were then observed for 48 hours. Mortality rates over this period were $10,20,60$, and $100 \%$ for animals given $10,20,40$, and $60 \mathrm{mg} / \mathrm{kg}$ of TAA, respectively, with virtually all deaths occurring 20-21 hours after administration of TAA. The dose of $40 \mathrm{mg} / \mathrm{kg}$ was chosen for subsequent studies as this was approximately the $\mathrm{LD}_{50}$.

Effect of pretreatment with EGF on TAA toxicity For logistical reasons, two independent experiments were performed on two separate occasions. In each experiment, mice (10 per group) were assigned randomly to receive saline or hrEGF (10 or $30 \mu \mathrm{g} / \mathrm{kg}$ intraperitoneally) 30 minutes before receiving TAA $(40 \mathrm{mg} / \mathrm{kg}$ intraperitoneally in a volume of $1 \mathrm{ml}$ ). After 24 hours, surviving animals were anaesthetised lightly with diethylether and blood collected via cardiac puncture. Animals were then killed by cervical dislocation and autopsies performed. Stomachs were collected and inflated with $3 \mathrm{ml}$ of $10 \%$ buffered formalin. They were subsequently opened along the greater curvature, rinsed in saline, and examined for mucosal damage using a dissecting microscope. The small intestinal tract was dissected free, flushed with $2 \mathrm{ml}$ of normal saline, weighed, opened along its length using dissecting forceps, and macroscopically inspected. The proximal $5 \mathrm{~cm}$ of the small intestine ("duodenum") was collected for analysis and a further five $(2 \mathrm{~cm})$ equi-spaced segments were then taken from the remaining length of the small intestine, fixed in buffered formalin, and subsequently processed for morphological assessment (see below). The kidneys, liver, large intestine, heart, lungs, spleen, and pancreas were also collected and processed for histological assessment.

A further six mice, housed under identical circumstances, were not given TAA or EGF but were treated in an identical fashion to determine locally validated "normal" values.

\section{MICROSCOPIC ANALYSES}

All histological and morphometric determinations were performed in a blinded fashion. Computer based image analysis was used to quantify small intestinal and hepatic damage. Briefly, microscopic images were captured using a Sony DXC-107 camera, displayed on an IBM computer via a VideoBlaster SE-100 frame grabber card. A $4 \times$ objective lens was used for assessment of intestinal injury and a $25 \times$ lens for hepatic assessment.

For intestinal injury, each microscopic field extended over $1500 \mu \mathrm{m}$ and included approximately 25 villi and vertically sectioned crypts. The entire length of the tissue samples was analysed using the DIGIPAT morphometric image processing package (EICISOFT Calle 24 No. 408, Vedado, Havana, Cuba). The total length of severely damaged mucosa, defined as regions of frank necrosis, was then determined and expressed as a percentage of the whole length of tissue examined.

Hepatic tissues were treated in a similar fashion with the images being captured and quantitated using the same equipment and software as that used for intestinal tissue. The total area of injury in each fragment was determined in a standardised tissue area of $40 \mathrm{~mm}^{2}$, by measuring and adding the size of the individual pericentral pale foci.

For renal tissue, the number of damaged glomeruli and haemorrhagic foci per field were determined using a $10 \times$ objective lens.

Effect of EGF on TAA toxicity when administered after TAA

To examine the potential value of EGF when given after TAA, two separate independent experiments were performed using identical protocols with $\mathrm{n}=10$ per group on each occasion. The protocol used was as described above except that EGF was given 30 minutes after TAA instead of 30 minutes before. For these studies, we also used a different set of parameters to measure gut injury to show that the apparent alteration in injury was not dependent on using a particular assay method. At the end of the study, the small intestine was flushed with $2 \mathrm{ml}$ of ice cold saline and the fluid collected for subsequent haemoglobin assay using a commercial kit (Reactivos SPINREACT SA, Barcelona, Spain) with the cyanomethahaemoglobin method. The small intestine was opened along its length, washed with further cold saline, blotted with paper, and weighed. It was then homogenised in phosphate buffered saline and aliquots taken to determine total protein using the Lowry method. Separate aliquots were used to determine total DNA content using a commercial kit (GeneQuant, Pharacia). Briefly, this was achieved by digesting the tissue homogenate 
Table 1 Twenty four hour survival values for rats given thioacetamide (TAA) $(40 \mathrm{mg} / \mathrm{kg})$ with or without epidermal growth factor (EGF) 30 minutes prior to administration of TAA. Ten mice were entered into each group on both experimental occasions

\begin{tabular}{lrrl}
\hline Experiment & TAA alone & $\begin{array}{l}\text { TAA+EGF } \\
(10 \mu g / k g)\end{array}$ & $\begin{array}{l}\text { TAA+EGF } \\
(30 \mu g / k g)\end{array}$ \\
\hline 1 & $4 / 10(40 \%)$ & $8 / 10(80 \%)$ & $10 / 10(100 \%)$ \\
2 & $6 / 10(60 \%)$ & $8 / 10(80 \%)$ & $10 / 10(100 \%)$ \\
Pooled data & $10 / 20(50 \%)$ & $16 / 20(80 \%)$ & $20 / 20(100 \%)^{\star}$ \\
\hline
\end{tabular}

${ }^{\star} \mathrm{p}<0.05 v$ TAA alone.

Table 2 Twenty four hour survival values for rats given thioacetamide (TAA) with or without epidermal growth factor (EGF) 30 minutes after administration of TAA. Ten mice were entered into each group on both experimental occasions

\begin{tabular}{lccc}
\hline Experiment & TAA alone & $\begin{array}{l}T A A+E G F \\
(10 \mu g / k g)\end{array}$ & $\begin{array}{l}T A A+E G F \\
(30 \mu g / k g)\end{array}$ \\
\hline 1 & $4 / 10(40 \%)$ & $7 / 10(70 \%)$ & $9 / 10(90 \%)$ \\
2 & $5 / 10(50 \%)$ & $8 / 10(80 \%)$ & $10 / 10(100 \%)$ \\
Pooled data & $9 / 20(45 \%)$ & $15 / 20(75 \%)$ & $19 / 20(95 \%)^{\star}$ \\
\hline
\end{tabular}

${ }^{\star} \mathrm{p}<0.05 v$ TAA alone.

for four hours at $55^{\circ} \mathrm{C}$ in extraction buffer containing $1 \mathrm{M}$ Tris pH 8, $0.5 \mathrm{M}$ EDTA, $20 \%$ SDS, and $20 \mathrm{mg}$ proteinase $\mathrm{K}$. This was followed by three cycles of phenol/chloroform extraction and the DNA precipitated with ethanol. The pellet was dried and resuspended in $100 \mu \mathrm{l}$ of TE for subsequent spectrophometric quantitation at $260 \mathrm{~nm}$. Results are expressed as the mean of three reading. Blood samples were collected by cardiac puncture for ALAT and creatinine assays.

STATISTICS

Data from the experiments using the same protocols on the two separate occasions were virtually identical, confirming the reproducibility of our findings. To demonstrate this reproducibility, we have shown the results for

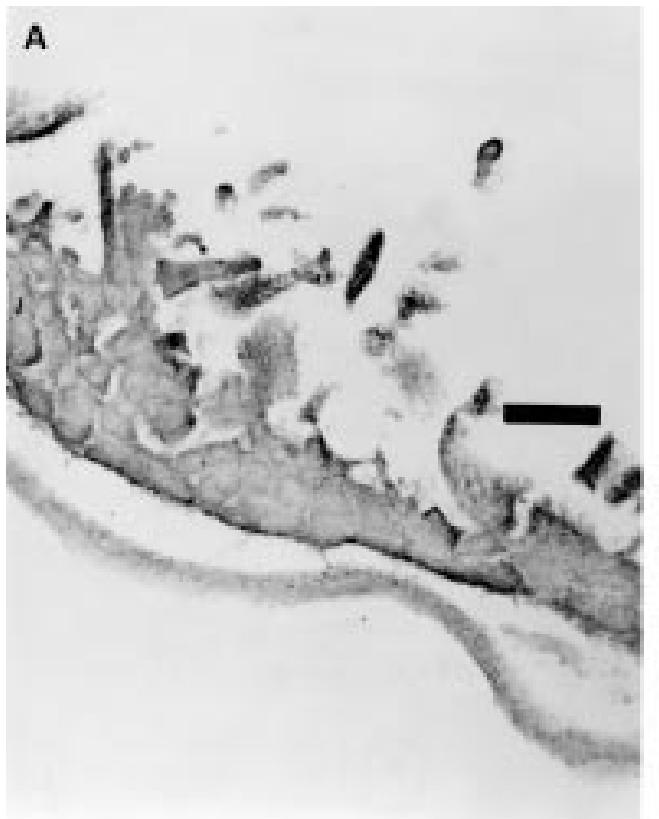

mortality data both as separate and cumulative results (tables 1,2). Subsequent analyses of data for organ damage from the two occasions have been pooled for statistical comparisons. Mortality data were compared using a $\chi^{2}$ test. As the data from biochemical and histological analyses were not normally distributed, data are presented as medians and interquartile ranges and statistical analyses used the nonparametric Mann-Whitney U test.

\section{Results}

MORTALITY

In animals who died within the initial 24 hour observation period, deterioration in general condition was observed 16 hours after TAA administration with progressive lethargy, piloerection, and prostration. All deaths occurred 20-21 hours after TAA injection. For the study involving animals that received EGF prior to TAA, mortality was $50 \%$ in control animals, $20 \%$ in animals that had received the lower dose of EGF, and $0 \%$ in animals given the higher dose of EGF (table 1). EGF showed a similar protective effect in animals when administered after TAA (table 2). Postmortem examination showed large amounts of intraluminal bleeding in the small intestine with histological changes similar to those found in animals that survived. Morphometric analyses of small intestinal tissue were not performed in animals that died in this initial 24 hour period as postmortem changes may have influenced the results.

MACROSCOPIC OBSERVATIONS OF SURVIVING ANIMALS AT AUTOPSY

Control animals showed increased friability of the small intestine and liver. Many of the animals also showed evidence of intraluminal

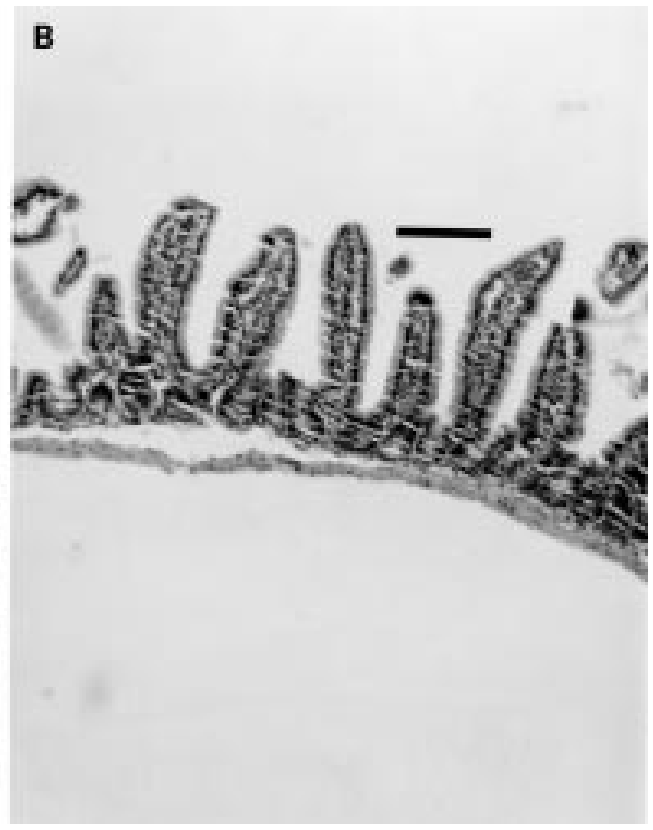

Figure 1 Thioacetamide (TAA) induced small intestinal injury. (A) Twenty four hours after administration of TAA (40 $\mathrm{mg} / \mathrm{kg}$ ) to mice, the normal villus structure of the jejunum and ileum is lost and marked necrosis is seen within the remaining mucosa. (B) Administration of epidermal growth factor (EGF, $30 \mu \mathrm{g} / \mathrm{kg}$ ), 30 minutes prior to TAA, markedly preserved small intestinal architecture. Similar preservation was seen when a dose of $10 \mu \mathrm{g} / \mathrm{kg}$ of EGF was used (not shown). Tissues were stained with haematoxylin and eosin. Original magnification $\times 10$. Bar $=100 \mu \mathrm{m}$. 
Table 3 Effect of pretreatment with epidermal growth factor (EGF, 10 or $30 \mu \mathrm{g} / \mathrm{kg}$ ) on thioacetamide (TAA) damaged organ systems. Control animals did not receive TAA or EGF. All of the reported parameters for the TAA treated groups (TAA alone and TAA+EGF) were significantly greater than those of the control

\begin{tabular}{|c|c|c|c|c|c|}
\hline \multirow[b]{2}{*}{ Organ } & \multirow[b]{2}{*}{ Parameter (units) } & \multicolumn{4}{|l|}{ Treatment } \\
\hline & & Control & $T A A$ & $T A A+E G F 10$ & $T A A+E G F 30$ \\
\hline Jejunum + ileum & Length showing necrosis ( $\%$ total) & $0(0-0)$ & $100(100-100)$ & $20.5(12.8-36.4)^{\star}$ & $10.1(8.5-15.8)^{\star}$ \\
\hline Liver & Area of necrosis (\%total) & $0(0-0)$ & $23.5(20.5-26.5)$ & $25(20.5-29.5)$ & $21.5(20-27)$ \\
\hline Liver & Serum ALAT (U/1) & $157(150-172)$ & $1550(1310-1790)$ & $1510(1165-1660)$ & $1710(1640-1760)$ \\
\hline Kidney & Serum creatinine $(\mathrm{mg} / 1)$ & $26(8.9-26)$ & $59(44-74)$ & $49.6(40.2-55.2)$ & $44(36.5-51.5)^{\star}$ \\
\hline Kidney & No collapsed glomerular tufts (per 10 high powered fields) & $0(0-0)$ & & $17.1(12.1-20)^{\star}$ & $8.6(5.7-13.6)^{\star}$ \\
\hline Kidney & No haemorrhagic foci (per 10 high powered fields) & $0(0-0)$ & & $2.1(1.1-3.9)^{\star}$ & $7.1(5.7-11.4)^{\star}$ \\
\hline
\end{tabular}

Results are median (interquartile range).

${ }^{\star} \mathrm{p}<0.05 v$ values in animals given TAA without EGF

bleeding into the small intestine. Similar changes were seen in the EGF treated animals although they were considered to be less severe qualitatively and this was confirmed quantitatively in animals given EGF after TAA (see below). Heart, lungs, spleen, pancreas, and kidneys appeared macroscopically normal.

FURTHER RESULTS FROM STUDIES WHERE EGF WAS GIVEN BEFORE TAA

Microscopic assessment

The major histological changes were present in the small intestine, liver, and kidney.

Intestine. Control animals had minimal histological changes in the duodenum. In contrast, the entire length of the jejunum and ileum showed extensive mucosal necrosis, usually associated with denudation (fig 1A). These changes were significantly reduced in both high and low dose EGF treated animals (fig 1B). Quantitative assessment showed that animals given TAA without EGF had mucosal necrosis of the jejunum and ileum affecting $100 \%$ of its length. In contrast, both doses of EGF significantly reduced the length of small intestinal tissue showing necrosis (both $\mathrm{p}<0.05 v$ TAA treated animals not given EGF; table 3). The large intestine was histologically normal in all groups.

Liver. Centrolobular necrosis, occasionally associated with haemorrhagic foci, were seen in all three groups (fig 2), often with some degree of inflammatory infiltration. Administration of

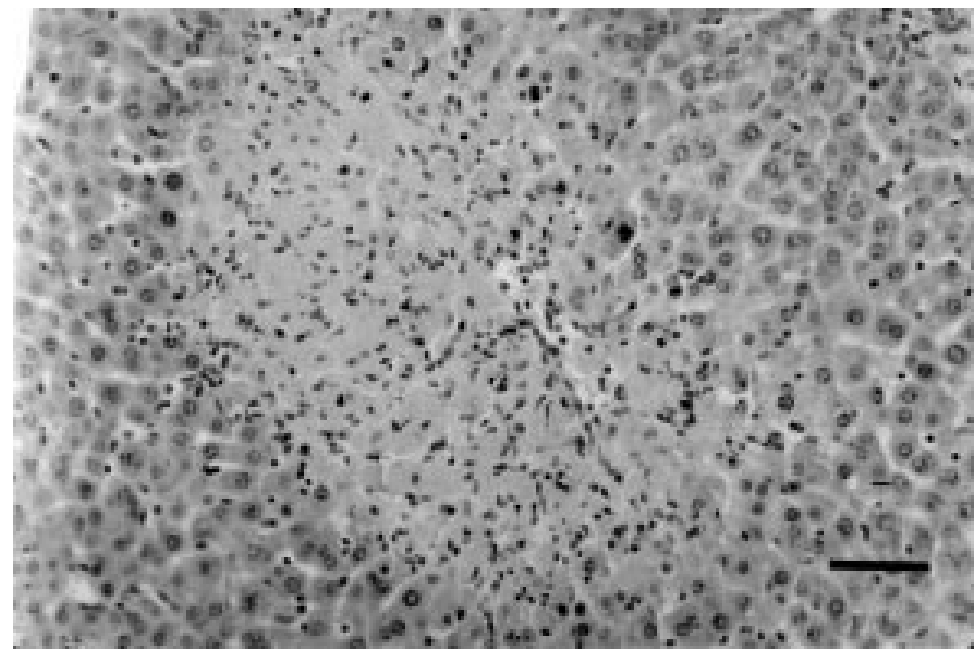

Figure 2 Thioacetamide (TAA) induced hepatic injury. TAA treated animals showed marked hepatic injury, mainly centrilobular necrosis with occasional haemorrhagic foci. The histological appearance was similar in all treatment groups. Tissues were stained with haematoxylin and eosin. Original magnification $\times 20$. Bar $=50 \mu \mathrm{m}$.
TAA caused serum ALAT levels to increase by about 10-fold ( $\mathrm{p}<0.01$ ) (table 3). Pretreatment with EGF did not affect the amount of hepatic injury caused in response to administration of TAA, as assessed using histology or the increase in transaminase levels (table 3 ).

Kidneys. Control animals showed extensive collapse of the glomerular tufts (fig 3A). Minor degenerative acute changes were also found in the epithelial cells of the tubular system and haemorrhagic areas were also present in the medulla (fig 3B). These changes were reduced in both high and low dose EGF treated animals (fig 3C). Quantitation, as assessed by the number of collapsed glomerular tufts and haemorrhagic foci seen during microscopic examination, and changes in plasma creatinine levels, supported this finding of a protective effect of EGF. For all of these parameters, administration of either the high or low dose of EGF resulted in a significant reduction in the amount of injury by about $70-80 \%$ (table 3 ).

FURTHER RESULTS FROM STUDIES WHERE EGF WAS GIVEN AFTER TAA

TAA alone caused a marked reduction in DNA and protein content expressed on a per gram wet weight basis (fig 4). TAA also caused a marked increase in luminal haemoglobin. As in the protocol involving EGF prior to TAA, plasma ALAT and creatinine increased. Administration of EGF after TAA reduced most of the changes caused by TAA (fig 4); luminal haemoglobin and plasma creatinine were significantly lower than in animals given TAA alone, and mucosal DNA and protein content were significantly higher. EGF did not, however, reverse the rise in ALAT caused by TAA.

\section{Discussion}

We examined the effect of administration of high dose thioacetamide on multiple organ systems and determined the influence of EGF on the degree of injury sustained. Administration of TAA ( $40 \mathrm{mg} / \mathrm{kg}$ intraperitoneally) was associated with $50 \%$ mortality with the major sites of injury being the small intestine, liver, and to a lesser extent the kidneys. Administration of EGF either before or 30 minutes after TAA markedly reduced the mortality rate, intestinal injury, and renal damage in a dose dependent manner. However, administration of EGF had little effect on the degree of hepatic injury sustained, as assessed histologically and biochemically.

Administration of TAA is a well established method of inducing hepatic injury. ${ }^{34}$ Its toxic- 

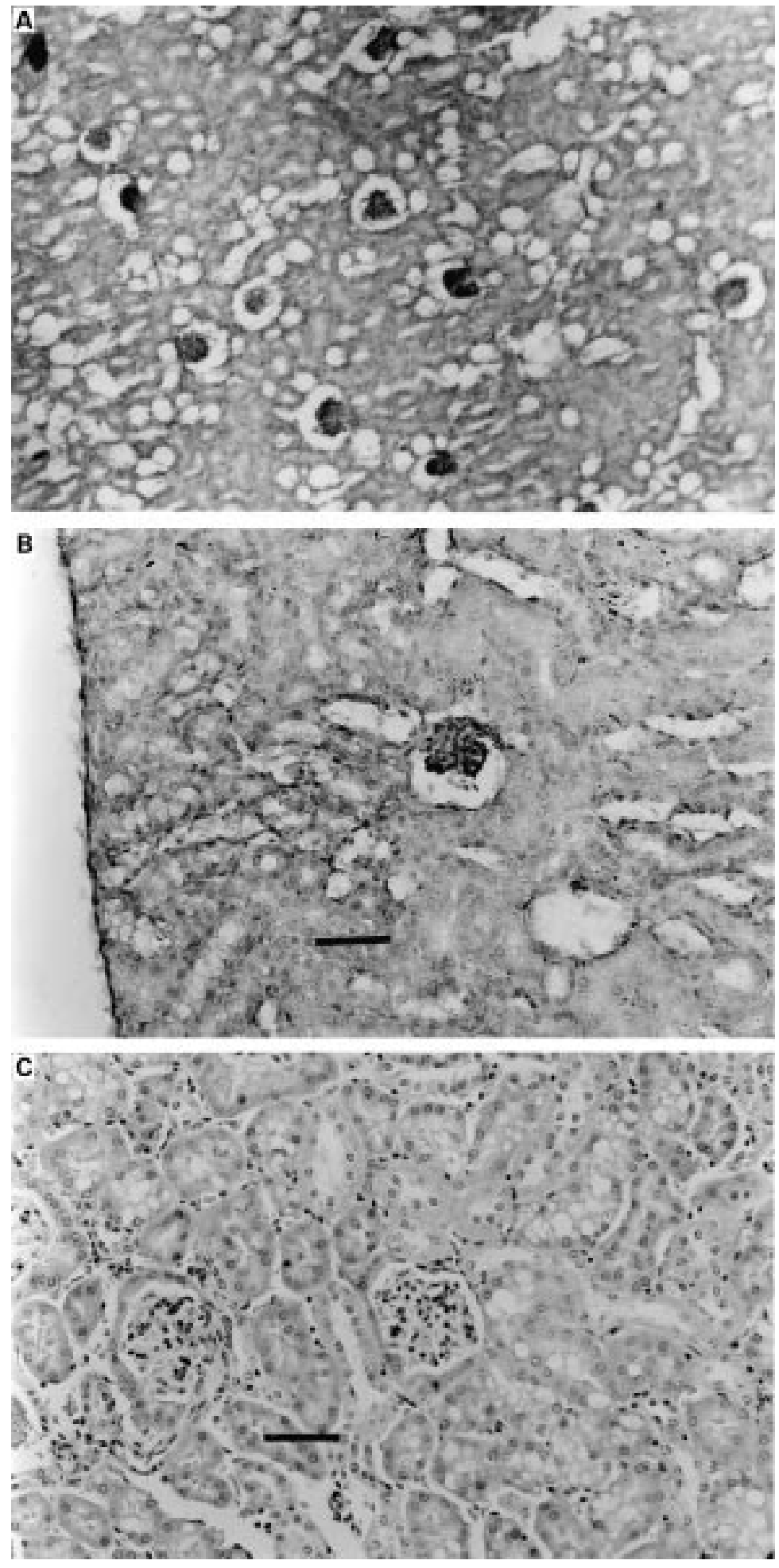

Figure 3 Thioacetamide (TAA) induced renal injury. Tissues were stained with haematoxylin and eosin. The renal tissue of TAA treated animals showed extensive collapse of the glomerular tufts.. Original magnification $\times 10$. (B) Higher power photomicrograph of the same tissue demonstrating that only minor degenerative acute changes in the epithelial cells of the tubular system were seen. Original magnification $\times 20$. Bar $=50 \mu \mathrm{m}$. (C)

Pretreatment with EGF reduced the renal changes caused by TAA. Original magnification $\times 20$. Bar $=50 \mu \mathrm{m}$. shown to be injured, including those present in the endoplasmic reticulum, those involved in mitochondrial respiration, and stress response proteins such as the heat shock protein family. ${ }^{6} 1213$ TAA may also injure cells by acting as a generator of free radicals, ${ }^{14}$ resulting in lipid peroxidation. Whatever the molecular mechanisms involved, administration of TAA results in increased apoptosis and necrosis, with necrosis being predominant when high doses of TAA are used. ${ }^{15}$ In the present series of studies, histological examination of intestinal tissue showed that necrotic changes were prominent throughout the small intestine. Necrosis was therefore likely to be the major final pathway of intestinal injury under these particular circumstances. Some degree of apoptosis does take place in this model, however, as shown by the presence of low levels of DNA laddering when collected intestinal residual DNA is run on agarose gels (CIGB, personal communication). Further examination of this process could potentially follow changes in apoptosis related enzymes such as caspases but would be complicated by the fact that little viable mucosal tissue is left in a field containing high levels of necrotic tissue.

The hepatic changes seen in the present study (centrilobular necrosis and raised transaminase levels) have been described previously. ${ }^{34}$ It has also been noted that TAA can injure other organ systems, including the intestine and kidneys. ${ }^{78}$ Ortega et al found that chronic oral administration of TAA to rats in their drinking water caused enlargement of intercellular spaces of the jejunum and ileum with lymphocytic infiltration. ${ }^{8}$ Much more extensive changes were found in our studies using mice where virtually complete necrosis of the mucosa was seen. This difference in toxicity probably relates to the dosage used (40 $\mathrm{mg} / \mathrm{kg}$ in the present study versus about 25 $\mathrm{mg} / \mathrm{kg}$ ) but may also relate to differences in the route of administration and species studied. In contrast with the study of Ortega et al, we examined the entire small intestine and found that the proximal gut (duodenum) was virtually unaffected by administration of TAA. The reason why the proximal bowel remained intact is unclear but may be due to enterohepatic circulation of toxic metabolites of TAA (which would result in relative sparing of the duodenum proximal to the ampulla), the requirement for pancreatic proteases to injure the intestine, or that the splanchnic blood flow to the duodenum was better preserved.

The predominant histological changes seen within the kidney were of tuft collapse with minor degenerative changes in the proximal tubule. Part of the explanation for this tuft collapse could be due to hypoperfusion induced by blood loss into the bowel lumen. Both the tuft collapse and tubular injury were prevented by administration of EGF. It is noteworthy that for both the intestinal and renal changes, TAA induced histological damage has been likened to that seen following ischaemia/reperfusion events. ${ }^{16}{ }^{17}$ In addition to the molecular mechanisms described above, TAA may therefore also cause injury by inducing vasoconstriction or by
TAA to the TAA sulphone and sulphoxide forms which react extensively with proteins, forming acetylimidolysine derivatives, resulting in their denaturation and charge modification. ${ }^{5}$ Multiple protein systems have been 

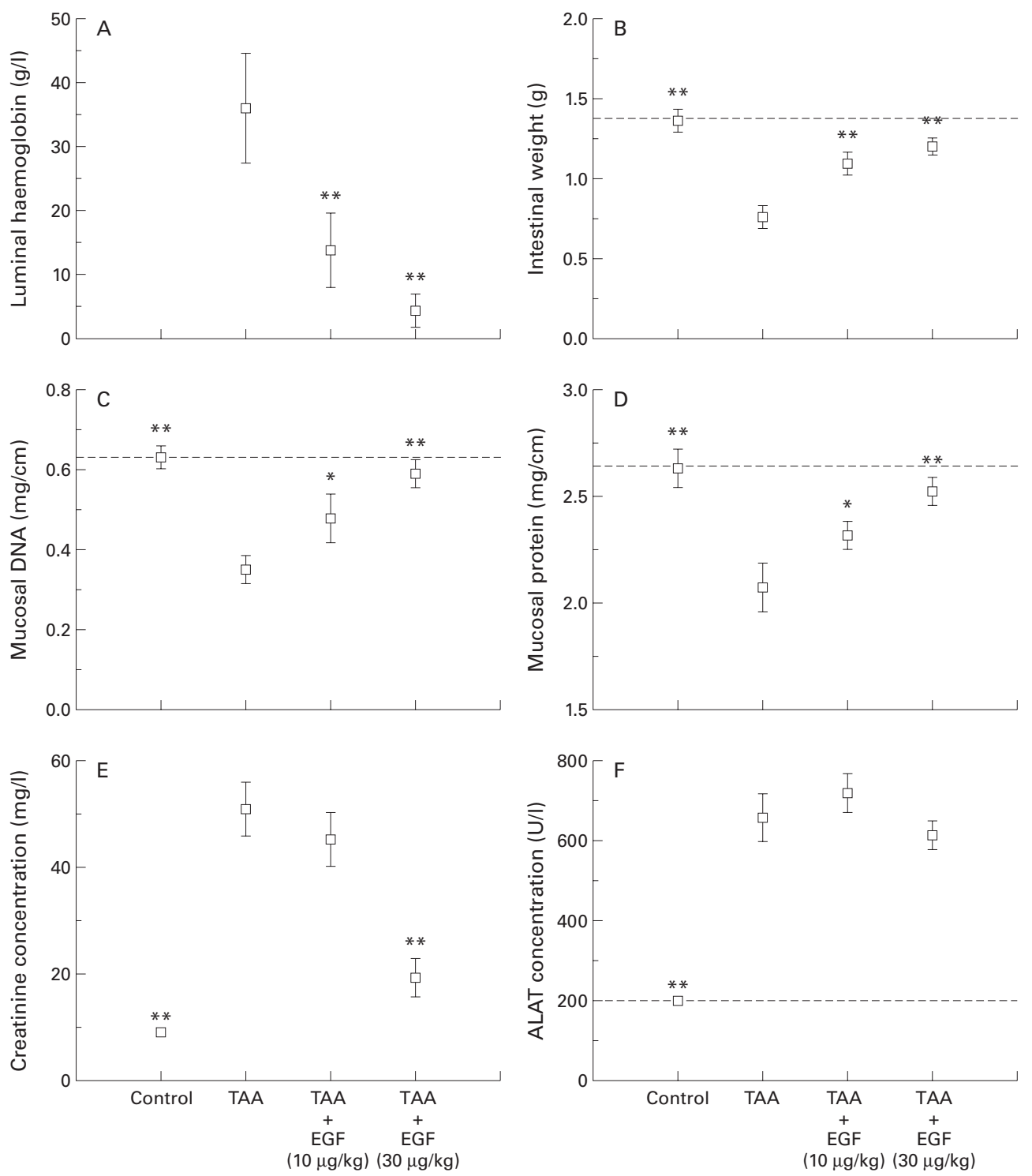

Figure 4 Effect of epidermal growth factor (EGF) given 30 minutes after thioacetamide (TAA) on multiple organ systems. EGF reduced luminal bleeding $(A)$, and the reduction in wet weight $(B), D N A(C)$, and protein content (D) of the gut caused by TAA. The rise in plasma creatinine caused by TAA was also reduced by administration of $E G F(E)$ although it did not influence the rise in serum alanine aminotransferase $(A L A T)(F) .{ }^{\star} p<0.05,{ }^{\star \star} p<0.01$ compared with animals given TAA alone.

interference with other key pathways involved in cellular respiration.

The major sources of EGF production are the salivary glands, Brunner's glands of the duodenum, and the kidney. Many studies have shown EGF to be a potent stimulant of growth for various cell types in vitro ${ }^{18}$ and in vivo ${ }^{19}$ and that it acts as a cytoprotective agent against gastrointestinal injury caused by a variety of noxious agents such as non-steroidal antiinflammatory drug induced gastric injury ${ }^{90}$ and trinitrobenzenesulphonic acid induced colitis. ${ }^{21}$ In addition, we have shown recently that exogenous EGF reduced carbon tetrachloride mediated hepatic damage, ${ }^{10}$ suggesting it might be of value in the prevention and treatment of hepatic injury. There is relatively little information, however, regarding its value for preventing/treating injury sustained in multiple organ systems, such as occurs during MOF.

The biochemical and histological data derived from these studies showed that EGF can markedly reduce intestinal and renal injury caused by TAA in a dose dependent fashion. The mechanism(s) by which EGF exerted these effects is, however, unclear. EGF influences multiple systems which might be important in mediating these effects, including upregulation of prostaglandin ${ }^{20}$ and mucus ${ }^{22}$ production, increasing mesenteric blood flow, ${ }^{23}{ }^{24}$ upregulation of superoxide dismutase (thereby reducing free radical induced toxicity), ${ }^{25}$ and influencing stress associated protein kinases. ${ }^{26}$ Interestingly, EGF did not appear to influence the degree of hepatic injury yet markedly diminished mortality. It is therefore likely that the severe intestinal damage with intraluminal haemorrhage, caused by TAA, was the major factor that resulted in death of the animals. 
Clinical trials of EGF are presently underway for the treatment of ulcerative conditions of the bowel, such as necrotising enterocolitis. ${ }^{27}$ Our studies provide preliminary evidence that EGF may also be of benefit in MOF where increased gut permeability is thought to be an important component in its aetiology. ${ }^{2} \mathrm{MOF}$ is a severe life threatening condition which usually occurs as a result of major trauma, burns, or fulminant infection. ${ }^{1}$ The pathophysiological mechanisms underlying $\mathrm{MOF}$ are unclear although important contributory factors probably include hypoxia, increased intestinal permeability, bacterial translocation, endotoxaemia, and uncontrolled systemic inflammatory responses. ${ }^{2}$ Whatever the initiating event, once established, MOF has a high mortality. ${ }^{1}$ Two of the most popular animal models used to induce MOF are administration of zymosan, ${ }^{28}$ which causes $\mathrm{MOF}$ by inducing pancreatic damage, and enterotoxin administration, which results in MOF via multiple mechanisms, including cardiovascular collapse. ${ }^{29}$ The present studies suggest TAA induced injury provides a complimentary model which has its predominant initial toxic effects on the liver and small intestinal mucosa.

Novel therapies that can reduce the risk of patients developing MOF have direct clinical potential. Many of the patients who progress to MOF while an inpatient can be identified at an early stage, often at the time of admission, based on the severity of initial injury, for example, percentage of skin surface area affected in burns patients. ${ }^{12}$ There is therefore a potential "therapeutic window" where agents such as EGF could be administered prior to the development of the full MOF syndrome. Further research examining the potential benefit of EGF itself or other EGF receptor ligands, such as transforming growth factor $\alpha$ and amphiregulin, in the prevention of MOF and in the treatment of MOF once fully established, appear warranted.

We thank Dr K O'Reilly, Department of Histopathology, for histological advice. Funding was provided by the Medical Research Council, Wellcome Trust, and Royal Society.

1 Zimmerman JE, Knuas WA, Sun X, et al. Severity stratification and outcome prediction for multisystem organ failure and dysfunction. World $\mathcal{F}$ Surg 1996;20:401-5.

2 Nguyen TT, Gilpin DA, Meyer NA, et al. Current treatment of severely burned patients. Ann Surg 1996;1:14-25.

3 Peeling J, Shoemaker L, Gauthier T, et al. Cerebra metabolic and histological effects of thioacetamideinduced liver failure. Am $\mathcal{F}$ Physiol 1993;28:G572-8.

4 Zimmermann T, Müller A, Machnik G, et al. Biochemical and morphological studies on production and regression of experimental liver cirrhosis induced by thioacetamide in Uje:Wistar rats. $Z$ Versuchstierkd 1987;30:165-80

5 Dyroff MC, Neal RA. Identification of the major protein adduct formed in rat liver after thioacetamide administration. Cancer Res 1981;41:3430-5.

6 Witzman FA, Fultz CD, Mangipudy RS, et al. Twodimensional electrophoretic analysis of compartment- specific hepatic protein charge modification induced by hioacetamide exposure in rats. Fundm Appl Toxicol 1996;31:124-32

7 Barker EA, Smuckler EA. Nonhepatic thioacetamide injury. II. The morphologic features of proximal renal tubular injury. Am f Pathol 1974;74:575-90.

8 Ortega MA, Torres MI, Fernandez MI, et al. Hepatotoxic agent thioacetamide induces biochemical and histological alterations in rat small intestine. Dig Dis Sci 1997;42:171523.

9 Playford RJ, Marchbank T, Calnan DP, et al. Epidermal growth factor is digested to smaller, less active forms in acidic gastric juice. Gastroenterology 1995;108:92-101.

10 Berlanga J, Caballero ME, Remirez D, et al. Epidermal growth factor protects against carbon tetrachloridegrowth factor protects against carbon tetra
induced hepatic injury. Clin Sci 1998;94:219-23.

11 Reitman S, Frankel S. A colorimetric method for the determination of serum oxalacetic and glutamic pyruvic transaminases. Am f Clin Pathol 1957;28:56-63.

12 Buko V, Ergov A, Karput S, et al. Mithocondrial respiration and oxidative phosphorylation in thioacetamide-induced liver necrosis. Toxicol Lett 1998;95(suppl 1):162.

13 Zimmermann T, Franke H, Dargel R. Biochemical and substructural studies on hepatic and serum lipoprotein metabolism after acute liver injury induced by thioacetamide in rats. Exp Pathol 1985;28:225-8.

14 Mesa ML, Carrizosa R, Martinez Honduvilla C, et al. Changes in rat liver gene expression induced by thioacetamide. Protective role of S-adenosyl-L-methionine by a gluthatione dependent mechanism. Hepatology 1996;23: 600-6.

15 Mangipudy RS, Rao PS, Andrews A, et al. Dose-dependant modulation of cell death: Apoptosis versus necrosis in thioacetamide hepatotoxicity. Int $\mathcal{f}$ Toxicol 1998;17:193211.

16 Parks AD, Bulkley GB, Granger DN, et al. Ischemic injury in the cat small intestine: Role of superoxide radicals. Gastroenterology 1982;82:9-15.

17 Racusen CL. The histopathology of acute renal failure. In: Ronco C, Bellomo R, eds. Critical care nephrology. Norwell, Ronco C, Bellomo R, eds. Critical care nephrology.

18 Conteas CN, Majumdar APN. The effects of gastrin, epidermal growth factor and somatostatin on DNA syntheis in a small intestinal crypt cell line (IEC-6). Proc Soc Exp Biol Med 1987;184:307-11

9 Goodlad RA, Lee CY, Wright NA. Cell proliferation in the small intestine and colon of intravenously fed rats: effects of urogastrone-epidermal growth factor. Cell Prolif 1992;25: 393-404.

20 Uribe JM, Barret KM. Nonmitogenic actions of growth factors: An integrated view of their role in intestinal physiology and pathophysiology. Gastroenterology 1997;112:25568.

21 Procaccino F, Reinshagen M, Hoffmann P, et al. Protective effect of epidermal growth factor in an experimental model of colitis in rats. Gastroenterology 1994:107:12-17.

22 Saroseik J, Bilski J, Murty VLN, et al. Role of epidermal growth factor in the maintenance of physico-chemical characteristics of oral and gastric mucus coat. Biochem Biophys Res Commun 1988;152:1421-7.

23 Gan BS, Hollenberg MD, MacCannel KL, et al. Distinct vascular actions of epidermal growth factor-urogastrone and transforming growth factor $\alpha$. F Pharm Exp Ther 1987; 242:331-7.

24 Tepperman BL, Soper BD. Effect of epidermal growth factor, transforming growth factor $\alpha$ and nerve growth factor on gastric mucosal integrity and microcirculation in the rat. Regul Pept 1994;50:13-21.

25 Price LT, Chen Y, Frank L. Epidermal growth factor increases antioxidant enzyme and surfactant system development during hyperoxia and protects fetal lungs in vitro from hyperoxic toxicity. Pediatr Res 1993;34:577-85.

26 Logan SK, Falasca M, Hu P, et al. Phosphatidylinositol 3-kinase mediates epidermal growth factor-induced activation of the c-jun N-terminal kinase signaling pathway. $\mathrm{Mol}$ Cell Biol 1997;1 7:5784-90.

27 Sullivan PB, Lewidon PJ, Oppenheimer SJ, et al. A pilot study of the safety and efficacy of epidermal growth factor in the treatment of necrotising enterocolitis. Gut 1997; 41(suppl 3):A69.

28 Jansen MJ, Hendriks T, Verhofstad AA, et al. Gradual development of organ damage in the murine zymosan-induced multiple organ dysfunction syndrome. Shock 1997;8:261 7 .

29 Wray GM, Hinds CJ, Thierman C. Effects of inhibitors of poly(ADP-ribose) synthetase activity on hypotension and
multiple organ dysfunction caused by endotoxin. Shock 1998;10:13019. 